\title{
The Relationship Between Olfactory Function and Emotional Contagion
}

\author{
Alexander W. J. Freemantle ${ }^{1}$ (D) $\cdot$ Lorenzo D. Stafford $^{1} \cdot$ Christopher R. D. Wagstaff $^{2} \cdot$ Lucy Akehurst $^{1}$
}

Received: 28 April 2021 / Accepted: 9 September 2021 / Published online: 23 September 2021

(c) The Author(s) 2021

\begin{abstract}
Introduction Research has provided evidence for the transfer of single emotions including anger, anxiety and happiness through olfactory chemosignals, yet no work has examined the role of odour function in the aggregation of more complex emotional states or in the emotional contagion process. The aim of the present study was to ascertain whether an individual's tendency to experience emotional aggregation was affected by objective measures of their olfactory function and subjective self-assessments of the importance of their own olfactory system.

Methods In this study $(N=70)$, participant pairs were first assessed individually for olfactory threshold and odour identification, then completed the Importance of Olfaction Questionnaire. Each pair subsequently took part in two collaborative tasks. Individual emotion measures were taken before, during and after the completion of the two tasks.

Results Multilevel structural equation modelling revealed that individuals' within-dyad positive emotional agreement scores were associated with both their 'importance of olfaction' scores and their olfactory function. A significant association was also found between olfactory performance and the Importance of Olfaction scores.

Conclusions These results provide evidence that the subjective importance an individual assigns to their sense of smell can predict their susceptibility to experience emotional aggregation during active, collaborative tasks.

Implications The findings suggest that individuals' tendency and capability to detect and respond to emotional chemosignals, a process required for olfactory-facilitated emotional contagion, may be affected by individual differences in olfactory function and subjective attitudes toward olfaction.
\end{abstract}

Keywords Emotional contagion $\cdot$ Chemosignals $\cdot$ Threshold detection $\cdot$ Odour identification $\cdot$ Olfaction importance

Social communication can be facilitated through visual, auditory and olfactory sensory modalities. Indeed, olfaction has been shown to possess multiple advantages

Alexander W. J. Freemantle

Alexander.freemantle@ port.ac.uk

Lorenzo D. Stafford

Lorenzo.stafford@port.ac.uk

Christopher R. D. Wagstaff

Chris.wagstaff@port.ac.uk

Lucy Akehurst

Lucy.akehurst@port.ac.uk

1 Department of Psychology, University of Portsmouth, Portsmouth PO1 2DT, UK

2 School of Sport, Health and Exercise Science, University of Portsmouth, Portsmouth PO1 2DT, UK for communication (Pause 2012). To elaborate, the social communication benefits of the olfactory system exist, in part, as a result of the production and detection of chemosignals. Human chemosignals can be found in bodily secretions such as tears (Gelstein et al. 2011) and sweat (Chen \& Haviland-Jones 2000) and can relay information relating to an individual's age, health, gender, social connection and emotional state (de Groot et al. 2017; Lubke \& Pause 2015). Seminal research undertaken by Chen and Haviland-Jones (2000) provided evidence that the experience of different emotions can cause the production of identifiable chemosignal profiles. Chen and Haviland-Jones also showed that these emotionally descriptive chemosignals could be detected, identified and differentiated using the olfactory system. Moreover, recent research has shown that the detection of these emotionrelated chemosignals can cause a subsequent change in a detector's emotional state in response to a donor's emotional 
state (see de Groot et al. 2017). In the current research, we sought to examine the role of individual differences (i.e. olfactory function, consisting of olfactory threshold and identification, and individuals' perceived importance of olfaction attitudes) in the olfactory-facilitated emotional aggregation process. Identifying individual differences in the detection and processing of olfactory emotional chemosignals will allow for a more detailed explanation regarding the mechanisms by which these chemosignals can influence other individuals. This greater understanding will assist researchers in examining the concept and provide a basis for real-world manipulation.

\section{Olfactory Function}

The detection of stimuli containing either social or nonsocial odours requires processing within the primary level of the olfactory system (de Groot et al. 2012; Tirindelli et al. 2009). One useful index of this detection is the use of olfactory threshold sensitivity tests. Olfactory sensitivity is related to a broad range of neurological processes including eating behaviour (Palouzier-Paulignan et al. 2012) and emotion (Pollatos et al. 2007; Stafford and Welbeck 2011). For instance, for the latter, it has been shown that increases in negative mood are inversely related to olfactory sensitivity (Pollatos et al. 2007; Stafford and Welbeck 2011). Additional evidence has also been presented which has shown a relationship between individuals' olfactory performance and their aptitude for higher-order executive functioning (Hedner et al. 2010). Hedner et al.'s (2010) findings, coupled with the link between emotion and olfaction, raise the possibility that an individual's odour function may be found to impact upon the complex emotional chemosignalling process and thus an individual's susceptibility to experience emotional aggregation.

Haviland-Jones, McGuire and Wilson (2016) found that there existed three social odour detector groups (super-detectors, detectors and non-detectors) with regard to participants' accuracy in sorting donated odours into fear, happiness and control groups. These findings suggest that there may be a distribution of capability in identifying social odours in much the same way as individuals' non-social odour identification. Therefore, testing aspects of individuals' olfactory functioning, such as odour identification capabilities, could be an important facet in assessing their tendency to differentiate socioemotional odours. Yet, Haviland-Jones and colleagues' study did not examine participants' ability to detect and emotionally respond to implicit emotional chemosignals-those originating from odours that participants are not explicitly told are socially relevant. As a result, it is still unclear how individual odour detection functions in real-world situations when individuals are presented with a cloud of olfactory noise containing both social and nonsocial odours.

\section{Olfaction and Emotion Contagion}

The discrimination of socio-emotional odours, though important, is only one part of the jigsaw and links to the question of whether once detected, chemosignals could have an emotionally contagious effect upon receivers' emotional states. Evidence for the emotional contagion (the unconscious interpersonal transfer of mood and affect, Hatfield et al. 1994) of singular emotions, as facilitated by the olfactory system, has been demonstrated throughout the literature across varying affect and methodologies (see de Groot et al. 2017 for review). Studies which have investigated the transfer of fear and anxiety through olfaction have predominately involved presenting participants with body odours collected from the axilla of donors. Researchers induce donors to feel anxious using methods such as administering academic exams (Prehn et al. 2006) and asking participants to undertake 'high ropes' courses (Albrecht et al. 2011). The effect that the presented chemosignals have upon the participant receiver may then be assessed through the coding of fearful facial expressions (de Groot et al. 2012) and subjective emotion measures (Albrecht et al. 2011). In these laboratory studies, the odour is explicitly presented to the receiver and detection of the odour is as controlled as possible. Nevertheless, Singh et al. (2018) found that dental students' procedural performance was worse whilst simply working on a mannequin dressed in a t-shirt that an anxious donor had worn, when compared with their performance whilst the mannequin was wearing a non-anxious body odour t-shirt. Singh et al. suggested that the student dentists had detected, and were emotionally affected by, the donor body odour whilst in close proximity to the mannequin. Singh et al.'s findings show that an explicitly controlled proximity and odour presentation technique is not required for the olfactory detection of body odour and the identification of a subsequent emotionally contagious effect. Indeed, Singh et al. (2018) provided evidence for the potential role of olfactory-facilitated emotional communication in dayto-day human experiences and showed that body odours were communicatively relevant even when masked by other non-social odours (as they could be by deodorants or perfumes in real life).

In contrast to the negative emotion literature, the interpersonal transfer of positive emotional chemosignals remains understudied. This reduced attention may be due to the tendency to investigate the so called hot emotions (see Fredrickson 2003), despite the experience 
of positive emotion being associated with evolutionary benefits (Fredrickson 2001; Steptoe et al. 2005; Taylor et al. 2000), as well as advantages in social situations (Fredrickson 2003). Increased positive emotions have been shown to improve immune function (Cohen et al. 2003) and increase interpersonal trust (Dunn \& Schweitzer 2005). Hence, a greater examination of the interpersonal transfer of positive emotions is necessary. Within the chemosignalling literature, research has shown that the body odour of a donor experiencing happiness can cause receivers to exhibit happier facial expressions when measured using EMG (de Groot et al. 2015).

In sum, the extant evidence can be interpreted to indicate that both positive and negative emotional chemosignals can cause interpersonal emotional transfer and affect receivers' emotions. Researchers have also shown that this interpersonal effect can occur in real-life scenarios in which odour proximity and detection are not explicitly controlled. The relationship between emotion and olfaction raises the question as to whether an individual's odour sensitivity can impact upon complex emotional chemosignalling processes and thus an individual's susceptibility to experiencing emotion contagion.

\section{Perceived Importance of Olfaction}

Subjective attitudes toward olfaction may also affect individuals' tendency to detect and process social emotion-related chemosignals. For instance, the Importance of Olfaction Questionnaire (Croy et al. 2010; IOQ) measures individuals' attitudes toward the associations related to specific olfactory sensations and the tendency for one to use their sense of smell in daily social situations. Indeed, scores on the IOQ have been shown to correlate with social communicative factors such as an individual's tendency to exhibit socially conforming behaviours (Seo et al. 2013). Seo et al. presented evidence for a relationship between self-reported socially relevant personality traits with importance of olfaction scores. It was argued that the neuroanatomical convergence of olfactory and emotional brain areas was a possible explanation for this finding. Additionally, Sorokowska et al. (2018) suggested that individuals paying more attention to the odours that they detected explained the evidence they found indicating the existence of individual differences in self-reported social odour awareness. This increased olfactory consideration may consequently lead to others' positive or negative olfactory chemosignals being more readily detected. Furthermore, evidence has been presented which highlights a positive relationship between an individual's attitudes toward their olfaction and their olfactory performance (Landis et al. 2003a). In sum, it is suggested that measures of individuals' attitudes toward olfaction, such as the IOQ, may play an important role in how individuals experience social situations.

\section{A Mix of Emotion}

The methodology used in the majority of research in this area require donors to be induced into experiencing a specific intense emotion before their body odour is collected. Though an efficient methodology, this ignores the fact that human emotional experience is complex, featuring intertwining effect that is constantly fluctuating (Kuppens et al. 2010). The range of emotions that an individual may experience during these tasks will combine to form general emotional states which may, at times, offer additional relevant social information alongside any primary emotion. Yet, researchers using the donor-receiver methodology in single emotion transfer studies inadvertently ignore this emotional complexity. As well as during the intense situations created in chemosignalling research, wider emotional states are experienced frequently in everyday life. These more general affective experiences may not prompt a fight or flight response, but nevertheless have been shown to influence others (Van Kleef 2009) and support social communication (Lount 2010; Waugh $\&$ Fredrickson 2006). The frequency with which these emotional states are experienced, in addition to the social and evolutionary advantages, highlight the role that chemosignalling communication and the olfactory system may hold in their interpersonal transfer. Nonetheless, the role of olfactory mechanisms in the transfer of less intense, but commonly experienced, positive and negative general affective states has not yet been investigated.

\section{The Current Study}

The current study was conducted to move beyond the limitations of the sender-receiver model of odour donation/presentation and instead examined broader emotional aggregation in the chemosensory domain. The aim was to investigate the relationship between individual odour function (olfactory threshold sensitivity, olfactory identification), subjective measures of odour (Importance of Olfaction Questionnaire, IOQ) and the extent to which two partners' emotions converge. Hence, is it the case that an individual's odour function relates to their ability to experience emotional aggregation from their partner. We tentatively predicted that participants who exhibited higher odour function would achieve a higher emotional convergence with their partner. As this reflects the first time that the IOQ has been used in chemosignalling work, our examination of the relationship of IOQ was more exploratory in nature. 


\section{Materials and Methods}

\section{Participants}

Seventy participants (53 female and 17 male) were recruited to take part in this study. The participants' mean age was 19.4 years $(\mathrm{SD}=2.3$ years) and all participants reported being non-smokers and non-vapers. The participants were tested as 35 pairs of individuals known to each other (23 F/F dyads, 7 F/M dyads and 5 M/M dyads). Sample size was predetermined on the basis of recommendations for multilevel modelling ( $>30$ participant clusters, per Maas \& Hox 2005; Scherbaum \& Ferreter 2009).

An adapted version of the Perceived Interpersonal Closeness scale (Popovic et al. 2003) was utilised to assess the subjective closeness of each pair relationship. The scale used a semi-circle structure with six concentric rings moving away from a centre point. The ring labels ranged from Distant to Fully Close. The participants were told that 'feeling close' was defined as, being listened to, understood by, able to share feelings with, and to talk openly with their partner. Participants marked with a cross how close they felt they were to their partner. Perceived closeness was operationalised as the distance between this cross and the centre point. The mean perceived closeness of the participants with their partner was $3.61 \mathrm{~cm}$ $(S D=1.39)$ this equated to 'Very Close'. This closeness score was used as a control variable in the subsequent mediation model.

\section{Measures}

\section{Olfactory Threshold}

An olfactory threshold test was completed to measure the participants' olfactory threshold sensitivity. The odorant used in this threshold test was butanol which was diluted in deionised water. The odorant was prepared using sixteen amber bottles in 16 dilution steps starting at $0.125 \%$ (step 1), with each successive step diluted by a factor of 2 using serial dilution to the lowest (step 16). At each dilution step, the bottle containing the odour was accompanied by two 'blank' bottles containing only the dilutant. A widely used forced choice single up-down staircase method was utilised (see Hummel \& Welge-Luessen 2006). The threshold test was completed once there had been 7 turning points, with the mean of the last 4 turning points indicating a participant's threshold score. Each testing bottle was held $2 \mathrm{~cm}$ below a participant's nose and waved below both nostrils to ensure optimal inhalation. The participants were instructed to wear a blindfold so as to avoid any identification of the odour bottles, and the experimenter wore cotton gloves to reduce odour cross contamination.

\section{Olfactory Identification}

The Sniffin' Sticks Identification test (Hummel et al. 1997) was used to assess the participants' odour identification ability. The test consisted of 16 pens which each contained the smell of a household odour. For each pen, the cap was removed by the experimenter and the pen tip was placed approximately $2 \mathrm{~cm}$ below a participant's nostrils and waved left and right to ensure optimal inhalation across both nostrils. For each pen, the participants selected the odour they believed to be correct from a set of four response options.

\section{Importance of Olfaction Questionnaire}

The significance that the participants placed upon odour and their olfactory behaviours was measured using the Importance of Olfaction Questionnaire (Croy et al. 2010: IOQ). The IOQ consists of three subscales: application, association and consequence. The application subscale measures an individual's subjective belief regarding the extent to which they use their sense of smell in their daily lives (e.g. I smell my clothes to judge whether I have to wash them or not). The association subscale measures how important participants believe that odour is in evoking memories, emotions and values (e.g. certain smells immediately activate strong feelings). Finally, the consequence subscale represents the extent to which individuals believe that they use olfaction to make daily decisions (e.g. if my partner has a nasty smell, I avoid kissing them). Participants respond to each questionnaire item using a 4-point Likert scale ranging from 1 ( $I$ totally agree) to 4 (I totally disagree). The final IOQ score for each participant was calculated as the average of the three subscale scores, with high scores indicating that individuals regard the olfactory sense as having a large impact upon their lives.

\section{Positive and Negative Affect Scale}

The participants' emotional states were measured at four time points using the Positive and Negative Affect Scale (PANAS, McNair et al. 1971). This scale consists of 20 items, ten of which assess positive emotion and ten assess negative emotion. Participants responded on 5-point Likert scales $(1=\mathrm{Not}$ at all to $5=$ Extremely) regarding the extent to which they were experiencing the 20 emotions. Within-dyad emotional aggregation was measured by comparing either the positive emotion or negative emotion PANAS subscale scores for two members of the same dyad using an $r_{W G(J)}$ method. 


\section{Task Performance}

The participants completed two sub-tasks during the task phase of the study. First, the subjects played Nintendo Wii Sports tennis (Nintendo) on the same team as their partner for $10 \mathrm{~min}$; following this, the participants attempted to complete a jigsaw puzzle together for a further $10 \mathrm{~min}$. Performance in the Wii Sports tennis task was operationalised as the number of points won subtracted by the number of points lost. Performance during the jigsaw puzzle task was operationalised as the number of puzzle pieces that were correctly connected to at least one other puzzle piece. These two tasks were chosen as the partners were required to cooperate in order to succeed; in addition, the two tasks differed in that they were either physically active (Wii Sports) or physically passive (jigsaw puzzle). Both tasks have been used extensively within the literature (e.g. Carrasco et al. 2019; Fissler et al. 2017).

\section{Procedure}

The participants were instructed to arrive at the testing venue with a friend. On arrival, they were taken to separate rooms and each participant completed a PANAS emotion measure (time 1, the arrival time point) and the 'closeness to partner' rating scale. Following the completion of these measures, each participant undertook an odour threshold test, the odour identification test and the Importance of Olfaction Questionnaire. Once participants had completed these tests, they undertook a second PANAS emotion measurement in private (time 2 , the pre-task time point). The participants were then asked to come together for the collaboration tasks. They were briefly taught the Nintendo Wii gameplay controls and then played Wii Sports Tennis for $10 \mathrm{~min}$ on the same team against two computer-generated players. After playing for $10 \mathrm{~min}$, the participants were given another PANAS emotion measure to complete in their separate testing rooms (time 3 , the during task emotion measure). The participants then together attempted to complete as much of a jigsaw puzzle as possible for a further $10 \mathrm{~min}$. Following the second collaborative task, the participants were asked to complete a final PANAS emotion measure, again on their own (time 4, the post-task time point), before being debriefed and thanked for their time. These four emotion measurement time points were used as they each represented a different dyadic emotional significance. At the arrival time point, the two dyad members had not yet experienced any of the same events; at the pre-task time, the two partners had experienced the same olfactory testing procedures but separately from each other, then, during task and post-task times, the participants had experienced the same tasks together whilst working collaboratively. The study was approved by the institution ethics board (SFEC 2019-090) and all participants provided written informed consent.

\section{Statistical Analysis}

Repeated measures ANOVA analyses were carried out to assess the difference in emotional states across the four time points. The same technique was used to assess whether the emotion agreement scores significantly differed across time points. Intraclass correlation coefficients (ICC) were also calculated for both positive and negative emotion scores at all four data collection points using an Actor-Partner Interdependence Model (Kenny et al. 2006). ICC values present the extent to which participants' within-dyad emotion scores are similar when compared to the whole cohort's betweendyad emotion scores and thereby offer an index of emotional aggregation.

Multilevel Structural Equation Modelling mediation analyses were completed to ascertain whether the relationship between an individual's Sum Threshold and Identification (Sum TI) score and the extent to which their mood was aggregated with a partner (calculated using $r_{W G(J)}$ scores) was mediated by the subjective importance that olfaction has upon their lives. $\mathrm{R}_{W G(J)}$ agreement scores were calculated for positive and negative emotional states for all dyads at each time point using a tool developed by Biemann et al. (2012). These $\mathrm{r}_{W G(J)}$ agreement scores present an index of withindyad agreement among participant ratings of a single variable on multiple items (LeBreton et al. 2005), in this case, the agreement between a participants' assessment of their own emotional state with that of a dyad partner's assessment of their own emotional state. The individual participants were each nested into dyads alongside their known partner. The analysed multilevel structural equation mediation model took the level 1-1-2 format; therefore, the authors used an MPLUS (version 8.4) syntax outlined by Preacher et al. (2010). A maximum likelihood estimation method was used in order to adequately account for the non-normal distribution of the $\mathrm{r}_{W G(J)}$ agreement scores for negative emotion (Lai 2018). The syntax can be found in Appendix 24.

The mediation model was first investigated in relation to time 1 (arrival) and time 2 (pre-task) for both positive and negative emotional agreement. The individually rated subjective measure of partner closeness was controlled for in these instances. These four models did not meet the adequate model fit thresholds and the respective indirect $\mathrm{a} \times \mathrm{b}$ effects were non-significant.

The mediation model was run for the time 3 (during-task) and time 4 (post-task) time points after the pairs had had an opportunity to collaboratively complete the tasks and spend time with each other. A significant indirect effect would indicate that the relationship between olfactory threshold and identification scores with emotional aggregation values was 
Table 1 Descriptive statistics for each of the assessed olfactory measures

\begin{tabular}{lcc}
\hline Olfactory measure & Mean & SD \\
\hline Olfactory threshold score & 8.52 & 2.36 \\
Odour identification score & 12.07 & 1.84 \\
Sum threshold identification score & 20.59 & 3.30 \\
Importance of olfaction (IOQ) score $^{\text {Association subscale }}$ & 2.93 & .38 \\
Application subscale $^{\mathrm{a}}$ & 3.14 & .41 \\
Consequences subscale $^{\mathrm{a}}$ & 2.78 & .50 \\
\hline
\end{tabular}

Note: $\mathrm{N}=70$

${ }^{a}$ The three IOQ subscales are each listed here with the accompanying descriptive statistics

mediated by individuals' importance of olfaction score. The dyads' performance on the preceding task and the dyad partner closeness ratings were controlled for in the multilevel mediation model, so that any impact on the pairs' emotional agreement did not occur as a result of the partners' relationship or their collaborative performance.

\section{Results}

\section{Descriptive Statistics}

\section{Olfactory Sensitivity}

The descriptive statistics for the olfactory function tests can be found in Table 1. The olfactory threshold and identification variables were summed to create a Sum Threshold Identification score (Sum TI score) for each participant, a technique similar to previous research (e.g. Hummel et al. 2007). A Kolmogorov-Smirnov test indicated that the Sum TI scores were normally distributed $(D(70)=0.077, p=0.200)$.

\section{Perceived Importance of Olfaction}

Mean values for the Importance of Olfaction questionnaire and each of the three subscales are listed in Table 1 . The IOQ was found to have a Cronbach's alpha of 0.812 .

\section{Emotion Measures}

The participants' mean positive and negative emotion values can be found in Table 2, along with the repeated measures ANOVA results comparing the group differences. Positive and negative emotional states were significantly different across the four time points. Post hoc tests using the Bonferroni correction indicated that the mean score for participants' positive emotional state at time 3 was significantly higher than it was at time 1 , time 2 and time 4 . Post hoc Bonferroni tests for the negative emotional state ANOVA showed that the negative emotional state scores at time 1 were significantly higher than at time 2, time 3 and time 4 .

\section{Emotion Agreement}

The $\mathrm{r}_{W G(J)}$ scores for negative emotions across all four time points were shown to significantly differ using a Greenhouse-Geisser correction repeated measures ANOVA $\left(F(2.47,170.62)=4.11, p=0.01, \eta_{p}{ }^{2}=0.06\right)$. Following Bonferroni pairwise comparisons, it was found that negative emotion $\mathrm{r}_{W G(J)}$ scores significantly increased from time $1(M=0.899, S D=0.096)$ to time $3(M=0.942, S D=0.074)$. There were no significant differences between the $\mathrm{r}_{W G(J)}$ scores for positive emotion across the time points $(F(2.37$, $163.53)=1.03, p=0.37, \eta_{p}^{2}=0.02$ ).

The participants' negative emotion ICC scores were found to be significant at time 3 (ICC $=0.339, p=0.02$ ). In contrast, positive emotion ICC scores were significant at time 2, 3 and 4, although the ICC score was marginally higher at time 3 (Fig. 1). These findings indicate that participants' within-dyad emotional states were distinct from the whole sample's emotional states at time 3 , for negative emotions, and at time 2, 3 and 4, for positive emotions, therefore highlighting that within-dyad emotional aggregation had occurred at these time points.

\section{MSEM Mediation Model}

The MSEM mediation model investigating positive emotional agreement at time 3 (during task) presented adequate model fit $\left(\chi^{2}(3)=4.03, p=0.26, \mathrm{CFI}=0.93\right.$, TLI $=0.80$, RMSEA $=0.07)$. Sum TI significantly predicted individuals' IOQ scores $(B=0.121, p=0.003)$ such that individuals with better olfactory functioning reported that they relied upon their sense of smell more in everyday life. In terms of the positive emotion agreement scores, we found that these were not predicted by Sum TI score $(B=-0.138$, $p=0.140$ ), which was against our hypothesis. However, the emotion agreement scores were directly predicted by individuals' IOQ scores $(B=1.658, p=0.006)$, which was also qualified by an indirect effect of Sum TI for positive emotion agreement via IOQ score $(\mathrm{a} \times \mathrm{b} B=0.201, p=0.009)$. These findings suggest that the degree to which dyads' positive emotions converge is predicted by IOQ but less so by TI (Fig. 2). The control variables, preceding task performance and partner closeness, did not significantly predict positive emotion agreement. The same MSEM model was found to be non-significant for both positive and negative emotion agreement scores at time 4 and for negative emotion agreement scores at time 3 . 
Table 2 Descriptive statistics and differences between the experienced positive and negative emotion at time 1 , time 2 , time 3 and time 4

\begin{tabular}{|c|c|c|c|c|c|c|c|c|c|}
\hline \multirow[t]{2}{*}{ Emotion } & \multicolumn{2}{|c|}{ Time 1} & \multicolumn{2}{|c|}{ Time 2} & \multicolumn{2}{|c|}{ Time 3} & \multicolumn{2}{|c|}{ Time 4} & \multirow[t]{2}{*}{ Differences across time } \\
\hline & Mean & SD & Mean & SD & Mean & SD & Mean & SD & \\
\hline Positive Emotion & 2.94 & .61 & 2.94 & .63 & 3.31 & .78 & 3.01 & .82 & $F=18.94, p<.001$ \\
\hline Negative Emotion & 1.35 & .34 & 1.25 & .28 & 1.17 & .24 & 1.21 & .28 & $F=9.53, p<.001$ \\
\hline
\end{tabular}

Note: $N=70$
Fig. 1 A graph showing the intraclass correlation coefficient scores for positive and negative emotional states across the four data collection time points. These ICC scores were measured using an Actor-Partner Interdependence Model. Note. Higher ICC scores indicate a higher within-dyad emotional association

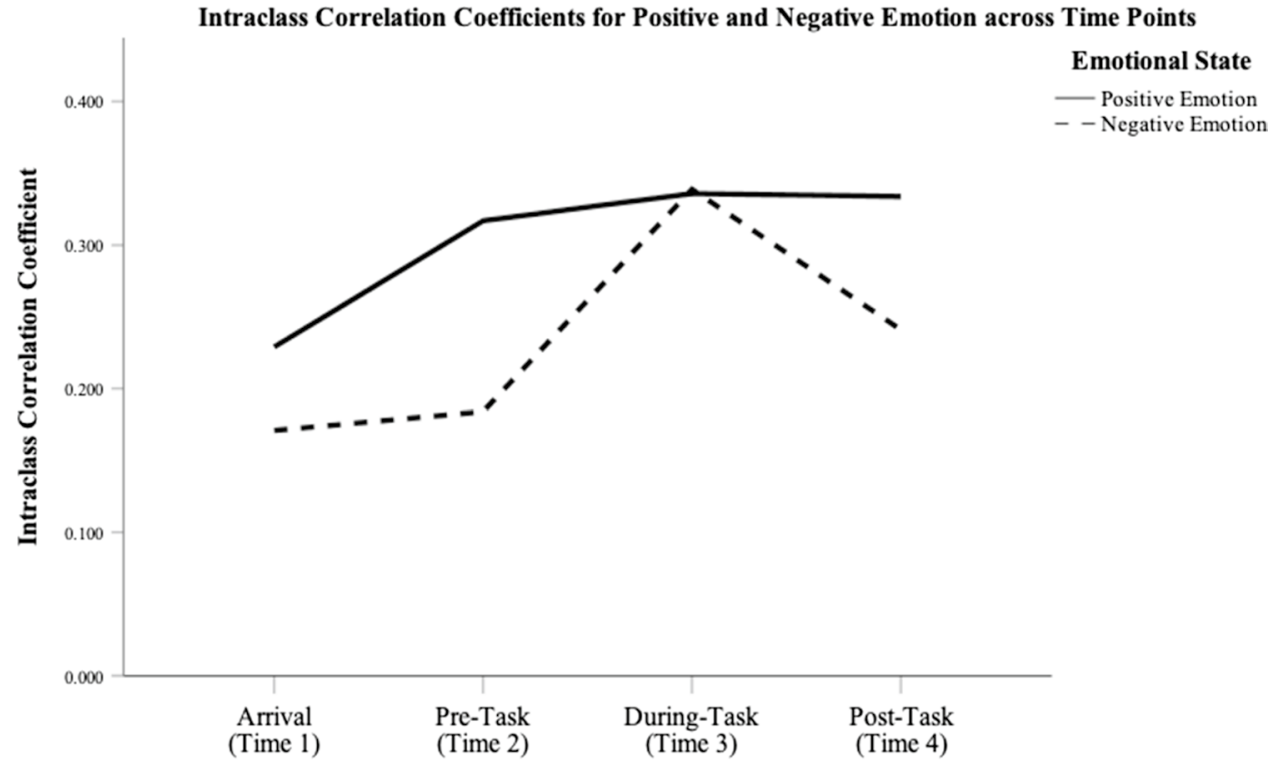

Note. Higher ICC scores indicate a higher within-dyad emotional association

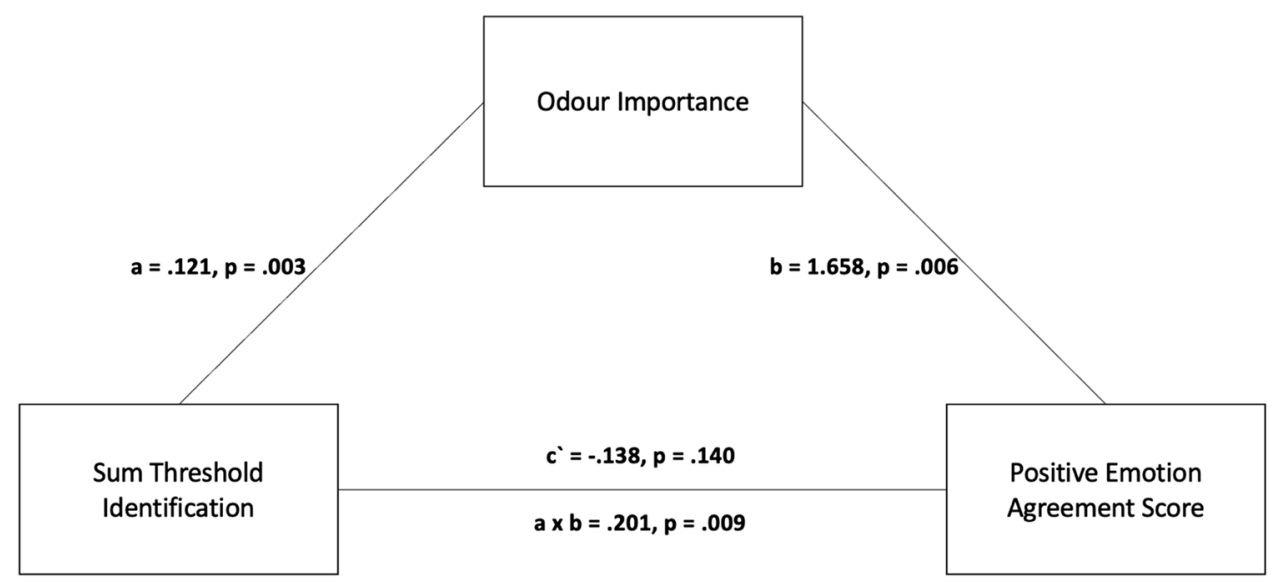

Fig. 2 The multi-level structural equation modelling mediation model

\section{Discussion}

In this study, we investigated the relationship between individuals' olfaction and their tendency to exhibit an aggregated emotional state to that of a collaborative partner. First, the participants were found to exhibit congruent within-dyad emotional states at three of the data collection time points, indicating that emotional aggregation 
had occurred. We further found a significant relationship between the participants' attitudes toward the importance of their olfaction (IOQ) and their olfactory performance (olfactory threshold and olfactory identification). These findings are unique and provide the first evidence that normosmic individuals' wider olfactory function may be associated with their attitudes toward olfaction. Evidence for a direct relationship between importance of olfaction measures and olfactory performance has previously been limited to one study (Landis et al. 2003a). Yet, Landis et al. did not use the Importance of Olfaction Questionnaire and only found evidence of a relationship between the importance of olfaction measure and odour identification. More recently, it was found that subjective attitudes toward olfaction (IOQ) were not related to objective odour function (Croy et al. 2011); however, in that study, IOQ was taken before odour function, whereas in the current study, the IOQ was completed after the olfactory tests. Relevant here is that previous work has identified that subjective assessments of olfactory ability can be positively associated with objective olfactory tests (Landis et al. 2003b), but only when the subjective estimation follows objective odour tests. To the authors' knowledge, the present study is the first to report a positive association between IOQ and odour function in normosmic individuals and also supports the work on estimating olfactory ability (Landis et al. 2003b) and the importance of test order. Moreover, it extends our understanding by showing that beyond estimating olfactory ability, one's broader attitude on the importance of olfaction is related to odour function.

We also found that the role of olfaction in the aggregation of emotion showed that dyads' positive emotional agreement scores were associated with both the individuals' importance of olfaction score (IOQ) (directly) and their sum threshold/ identification (TI) odour function score (indirectly via IOQ). The mediation model findings can be interpreted to suggest that the magnitude of emotional contagion between dyads may be associated with individuals' odour function, which supports and extends the literature. Research has demonstrated that emotions can transfer between individuals by means of olfactory-detected chemosignals (e.g. de Groot et al. 2012). Nevertheless, whereas previous work has focused on the transfer of specific emotions (e.g. anxiety; Dalton et al. 2013; Prehn et al. 2006), the present data show evidence for the transfer of more global emotional states which encompass several emotions. This is important as these complex emotional states are experienced frequently in social situations and their exhibition possesses unique social benefits, such as the building of interpersonal trust (Dunn \& Schweitzer 2005). The real-world utility of the findings is further highlighted through the ecologically valid methodology employed. In this study, the participant pairs completed collaborative tasks together in close proximity and the donor-receiver approaches often presented in the literature, and which require controlled explicit odour presentation techniques, were not utilised. The findings therefore suggest that the singular intense emotion, sender-receiver methodologies used in previous studies (e.g. de Groot et al. 2015) may not be required to investigate olfactory-facilitated emotional transfer. The benefits of using a more ecologically valid approach are clear, in that any findings can be interpreted to show evidence of olfactory-facilitated emotional contagion in a situational context mirroring a realworld scenario. Singh et al. (2018) showed that proximity to a socially relevant body odour was adequate to facilitate emotional contagion, and similarly, researchers have highlighted the role of olfaction and body odour detection in the natural formation of human social relationships (Mahmut and Croy 2019). Thus, the current study has provided further evidence that olfactory-assisted communication can be implicitly facilitated and can occur in applied, real-world environments. Prominent researchers encourage the completion of more ecologically valid human body odour research (Roberts et al. 2020), yet the authors recognise that there are some costs present in trading a controlled odour donation and presentation methodology for the methods used in this study, which are grounded in greater ecological realism. For instance, odour signals were not manipulated or measured and therefore the role of body odour in the identified within-dyad emotional convergence cannot be confirmed and an alternative factor may have affected the mediated relationship. Nevertheless, similar, more realistic methodologies have been utilised within the body odour literature (e.g. Frumin et al. 2015; Singh et al. 2018) and findings from the current study are similar to those of previous studies.

It was only at the third time point (during the collaboration tasks) that olfactory measures were found to influence the extent of emotional aggregation. There are two allied explanations for this; firstly, the Wii tennis task caused a significant change in positive emotion compared to the other time points and emotion intensity has been shown to be necessary in facilitating emotional contagion (Hatfield et al. 2014). Secondly, the Wii task involved more physical activity than the jigsaw puzzle task and more closely resembled tasks utilised in previously completed sweat odour donation studies (e.g. Mutic et al. 2016). Therefore, we theorise that the active Wii Sports task caused the production of emotionally communicative chemosignals (Lubke \& Pause 2015), which influenced participants' emotional aggregation. This explanation would provide further evidence for the role of olfactory mechanisms in the emotional convergence identified in this study.

In terms of odour function, it was surprising that there was a direct effect for the subjective measure of IOQ but not the objective measures of odour threshold and odour identification. Previous research has shown that odour threshold 
sensitivity is related to a number of emotional indices including disgust (Croy et al. 2017) and mood (Pollatos et al. 2007; Stafford and Welbeck 2011), and hence, a stronger association may have been expected in the present study. Nevertheless, it is important to note that those studies found evidence for the so-called hot emotions (disgust, negative mood) relating to threshold sensitivity, which contrasts with the present study where effects were observed only in positive emotional states. Similarly, Croy et al. (2017) and Stafford and Welbeck (2011) investigated participants' experienced emotions rather than their emotional aggregation, as was measured in the current study. It is therefore suggested that odour threshold and identification tests are less able to predict more complex social-emotional phenomena such as emotional aggregation. In contrast, the IOQ focuses more directly on the social aspects of individuals' olfaction and is therefore more able to capture the social-odour link. For instance, some of the IOQ items ${ }^{1}$ assess how an individual's sense of smell may impact upon their social interactions with others. This is also consistent with previous work which has found that individuals with higher IOQ scores tend to exhibit more socially conforming behaviours in social situations (Seo et al. 2013). Findings from the present study further indicate that the IOQ may better convey indications of an individual's social tendencies compared to objective TI measures. In addition, as has been suggested elsewhere (e.g. Smeets et al. 2008; Sorokowska et al. 2018), it could be that individuals who rate their sense of smell as important are also more aware of odours and consequently more sensitive to the positive and negative effects of odours. Extrapolating from this evidence, it can be theorised that individuals who score higher on the IOQ may rely more heavily on their sense of smell during social situations and therefore be more emotionally affected by others' positive and negative emotions. Hence, it is possible that such individuals are also more susceptible to emotional contagion. The findings from this study therefore highlight the first indication that subjective, socially relevant measures of olfaction, such as the IOQ, can be used to estimate an individual's tendency to exhibit an aggregated emotional state in social situations.

The investigated mediation model was not found to be significant during the transfer of negative affective states. This could be a result of the low instances of negative emotion experienced by participants in the current study. For instance, the participants' mean negative emotional state for the pre, during or post task time points did not exceed a score of 1.25 (using a Likert scale ranging from 0 Not at all-5 Extremely). Previously, it has been shown that the intensity

\footnotetext{
1 'The smell of a person plays a role in the decision as to whether I like him/her' and 'If my partner has a nasty smell, I avoid kissing them'.
}

of the experienced emotion is relevant in the interpersonal transfer of emotions (Hatfield et al. 2014). Thus, we consider that for the participants' experiences of negative emotion in the current study, this threshold was not met for specifically olfactory-facilitated emotional contagion to occur.

In terms of limitations, it could be contended that the odour tests would have been better utilised if the threshold test used social rather than non-social odours. Nevertheless, we note that the tests used here are standardised olfactory tests, used extensively in research (e.g. Hummel et al. 2007). Additionally, whilst related work has utilised synthesised social odours such as androstadienone (e.g. Lundstrom et al. 2006), it needs to be recognised that developing a threshold test for 'naturally' occurring social odours presents a number of technical challenges and would limit comparisons to future related work.

In conclusion, evidence has been presented here for the first time which indicates an association between Importance of Olfaction (IOQ) scores and normosmic individuals' odour function. Additionally, results from this study have shown that the importance that individuals assign to their sense of smell can predict their ability to experience an aggregate emotion. We posit that this relationship is caused by an increase in individuals' abilities to detect and respond to emotional chemosignals. Future research could further extend this work by continuing to examine the transfer of general affective states between individuals.

\section{Appendix 1}

MPLUS syntax for the 1-1-2 MSEM mediation model utilised in the present study.

USEVARIABLES ARE A B C D E F;

CLUSTER IS A;

BETWEEN ARE D E;

ANALYSIS: TYPE IS TWOLEVEL;

ESTIMATOR = MLR;

MODEL:

\%WITHIN\%

$\mathrm{C}$ ON B;

\%BETWEEN\%

$\mathrm{D}$ on $\mathrm{C}(\mathrm{b})$.

E

F

$\mathrm{B}$;

$\mathrm{C}$ on $\mathrm{B}(\mathrm{a})$;

MODEL CONSTRAINT:

$\mathrm{NEW}(\mathrm{ab})$;

$\mathrm{ab}=\mathrm{a}^{*} \mathrm{~b}$;

OUTPUT: TECH1 TECH8 CINTERVAL;

$\mathrm{A}=$ Dyad.

$\mathrm{B}=$ Sum threshold identification score. 
$\mathrm{C}=$ Importance of olfaction score.

$\mathrm{D}=\mathrm{r}_{W G(J)}$ positive emotion agreement scores (during-task time point).

$\mathrm{E}=$ Wii performance score.

$\mathrm{F}=$ Closeness to partner.

\section{Declarations}

Conflict of Interest The authors declare no competing interests.

Open Access This article is licensed under a Creative Commons Attribution 4.0 International License, which permits use, sharing, adaptation, distribution and reproduction in any medium or format, as long as you give appropriate credit to the original author(s) and the source, provide a link to the Creative Commons licence, and indicate if changes were made. The images or other third party material in this article are included in the article's Creative Commons licence, unless indicated otherwise in a credit line to the material. If material is not included in the article's Creative Commons licence and your intended use is not permitted by statutory regulation or exceeds the permitted use, you will need to obtain permission directly from the copyright holder. To view a copy of this licence, visit http://creativecommons.org/licenses/by/4.0/.

\section{References}

Albrecht J, Demmel M, Schopf V, Kleemann A, Kopietz R, May J, Schreder T, Zernecke R, Bruckmann H, Wiesmann M (2011) Smelling chemosensory signals of males in anxious versus nonanxious condition increases state anxiety of female subjects. Chem Senses 36:19-27

Biemann T, Cole MS, Voelpel S (2012) Within-group agreement: oOn the use (and misuse) of rwg and $\mathrm{rwg}(\mathrm{j})$ in leadership research and some best practice guidelines. Leadersh Q 23:66-80

Carrasco M, Ortiz-Maqués N, Martínez-Rodríguez S (2019) Playing with Nintendo Wii Sports: impact on physical activity, perceived health and cognitive functioning of a group of community-dwelling older adults. Act Adapt Aging 44(2):1-13

Chen D, Haviland-Jones J (2000) Human olfactory communication of emotion. Percept Mot Skills 91:771-781

Cohen S, Doyle W, Turner R (2003) Emotional style and susceptibility to the common cold. Psychosom Med 65:652-657

Croy I, Buschhuter D, Seo H, Negoias S, Hummel T (2010) Individual significance of olfaction: development of a questionnaire. Eur Arch Otorhinolaryngol 267:67

Croy I, Landis B, Meusel T (2011) Patient adjustment to reduced olfactory function. Archive of Otolaryngology Head and Neck Surgery 137(4):377-382

Croy I, Bendas J, Wittrodt N, Lenk M, Joraschky P, Weidner K (2017) Gender-specific relation between olfactory sensitivity and disgust perception. Chem Senses 42(6):487-492

Dalton P, Maute C, Jaen C, Wilson T (2013) Chemosignals of stress influence social judgements. PloS ONE 8(10):e77144

de Groot JH, Smeets MA, Kaldewaij A, Duijndam MJ, Semin GR (2012) Chemosignals communicate human emotions. Psychol Sci 23(11):1417-1424

de Groot J, Smeets M, Rowson M, Bulsing P, Blonk C, Wilkinson J, Semin G (2015) A sniff of happiness. Psychol Sci 26(6):684-700 de Groot J, Semin G, Smeets M (2017) On the communicative function of body odours: a theoretical integration and review. Perspect Psychol Sci 12(2):306-324

Dunn J, Schweitzer M (2005) Feeling and believing: the influence of emotion on trust. J Pers Soc Psychol 88(6):736-748

Fissler P, Küster OC, Loy LS, Laptinskaya D, Rosenfelder MJ, von Arnim CA, Kolassa IT (2017) Jigsaw Puzzles as Cognitive Enrichment (PACE) - the effect of solving jigsaw puzzles on global visuospatial cognition in adults 50 years of age and older: study protocol for a randomized controlled trial. Trials 18(1):415

Fredrickson B (2001) The role of positive emotions in positive psychology: the broaden-and-build theory of positive emotions. Am Psychol 56(3):218-226

Fredrickson B (2003) The value of positive emotions. Am Sci 91(4):330-335

Frumin I, Perl O, Endevelt-Shapira Y, Eisen A, Eshel N, Heller I, Shemesh M, Ravia A, Sela L, Arzi A, Sobel N (2015) A social chemosignalling function for human handshaking. Elife 3(4):e05154. https://doi.org/10.7554/eLife.05154

Gelstein S, Yeshurun Y, Rozenkrantz L, Shushan S, Frumin I, Roth Y, Sobel N (2011) Human tears contain chemosignals. Science 14(331):226-230

Hatfield E, Cacioppo J, Rapson R (1994) Emotional Contagion. Cambridge University Press

Hatfield H, Bensman L, Thornton P, Rapson R (2014) New perspectives on emotional contagion: a review of classic and recent research on facial mimicry and contagion. Interpersona 8(2):159-179

Haviland-Jones JM, McGuire TR, Wilson P (2016) Testing for individual differences in the identification of chemosignals for fear and happy: phenotypic super-detectors, detectors and nondetectors. PLoS ONE 11(5):e0154495. https://doi.org/10.1371/ journal.pone. 0154495

Hedner M, Larsson M, Arnold N, Zucco G, Hummel T (2010) Cognitive factors in odor detection, odor discrimination and odor identification tasks. J Clin Exp Neuropsychol 32(10):1062-1067

Hummel T, Welge-Luessen A (2006) Assessment of olfactory function. Advanced. Otorhinolaryngology 63:84-98

Hummel T, Sekinger B, Wolf S, Pauli E, Kobal G (1997) 'Sniffin' Sticks': olfactory performance assessed by the combined testing of odor identification, odor discrimination and olfactory threshold. Chem Senses 22:39-52

Hummel T, Kobal G, Gudziol H, Mackay-Sim A (2007) Normative data for the "Sniffin Sticks" including tests of odor identification, odor discrimination, and olfactory thresholds: an upgrade based on a group of more than 3,000 subjects. European Archive of Otorhinolaryngology 264(3):237-243

Kenny DA, Kashy DA, Cook WL (2006) Dyadic data analysis. Guilford Press

Kuppens P, Oravecz Z, Tuerlinckx F (2010) Feelings change: accounting for individual differences in the temporal dynamics of affect. J Pers Soc Psychol 99(6):1042-1060

Lai K (2018) Estimating standardized SEM parameters given nonnormal data and incorrect model: methods and comparison. Struct Equ Modeling 25(4):600-620

Landis BN, Giger R, Morel R, Lacroix JS (2003a) Auto-evaluation des performances olfactives. Swiss Med For 26(11):1154-1157

Landis BN, Hummel T, Hugentobler M, Giger R, Lacroix JS (2003b) Ratings of overall olfactory function. Chem Senses 28(8):691-694

LeBreton JM, James LR, Lindell MK (2005) Recent issues regarding $r_{W G}, r^{*}{ }_{W G}, r_{W G(J)}$, and $r^{*}{ }_{W G(J)}$. Organ Res Methods 8(1):128-138

Lount R (2010) The impact of positive mood on trust in interpersonal and intergroup interactions. J Pers Soc Psychol 98(3):420-433

Lubke K, Pause B (2015) Always follow your nose: the functional significance of social chemosignals in human reproduction and survival. Horm Behav 68:134-144 
Lundstrom J, Olsson M, Schaal B, Hummel T (2006) A putative social chemosignal elicits faster cortical responses than perceptually similar odorants. Neuroimage 30:1340-1346

Maas CJM, Hox JJ (2005) Sufficient sample sizes for multilevel modelling. Methodology 1(3):86-92. https://doi.org/10.1027/16141881.1.3.86

Mahmut MK, Croy I (2019) The role of body odors and olfactory ability in the initiation, maintenance and breakdown of romantic relationships - a review. Physiology and Behaviour 207:179-184. https://doi.org/10.1016/j.physbeh.2019.05.003

McNair DM, Lorr M, Droppleman LF (1971) Manual for the Profile of Mood States. Educational and Industrial Testing Services, San Diego, CA

Mutic S, Parma V, Brunner Y, Freiherr J (2016) You smell dangerous: communicating fight responses through human chemosignals of aggression. Chem Senses 41:35-43

Nesse R (1990) Evolutionary explanations of emotions. Hum Nat 1(3):261-289

Palouzier-Paulignan B, Lacroix MC, Aimé P, Baly C, Caillol M, Congar $P$ et al (2012) Olfaction under metabolic influences. Chem Senses 37(9):769-797

Pause B (2012) Processing of body odour signals by the human brain. Chemical Perception 5:55-63

Pollatos O, Kopietz R, Linn J, Albrecht J, Sakar V, Anzinger A et al (2007) Emotional stimulation alters olfactory sensitivity and odor judgment. Chem Senses 32(6):583-589

Popovic M, Milne D, Barrett P (2003) The scale of perceived interpersonal closeness (PICS). Clin Psychol Psychother 10:286-301

Preacher K, Zyphur M, Zhang Z (2010) A general multilevel SEM framework for assessing multilevel mediation. Psychol Methods 15(3):209-233

Prehn A, Ohrt A, Sojka B, Ferstl R, Pause BM (2006) Chemosensory anxiety signals augment the startle reflex in humans. Neurosci Lett 394(2):127-130. https://doi.org/10.1016/j.neulet.2005.10.012

Roberts C, Havlicek J, Schaal B (1800) (2020), Human olfactory communication: current challenges and future prospects. Philo Trans B: Biol Sci 375:20190258. https://doi.org/10.1098/rstb.2019.0258
Scherbaum CA, Ferreter JM (2009) Estimating statistical power and required sample sizes for organizational research using multilevel modelling. Organ Res Methods 12(2):347-367. https://doi.org/10. 1177/1094428107308906

Seo H, Lee S, Cho S (2013) Relationships between personality traits and attitudes towards the sense of smell. Front Psychol 4:1-8

Singh PB, Young A, Lind S, Cathinka Leegaard M, Capuozzo A, Parma V (2018) Smelling anxiety chemosignals impairs clinical performance of dental students. Chem Senses 43:411-417. https:// doi.org/10.1093/chemse/bjy028

Smeets M, Schifferstein H, Boelema S, Lensvelt-Mulders G (2008) The Odor Awareness Scale: a new scale for measuring positive and negative odor awareness. Chem Senses 33:725-734

Sorokowska A, Groyecka A, Karwowski M, Frackowiak T, Lansford J, Ahmadi K et al (2018) Global study of social odor awareness. Chem Senses 43:503-513

Stafford LD, Welbeck K (2011) High hunger state increases olfactory sensitivity to neutral but not food odors. Chem Senses 36(2):189-198

Steptoe A, Wardle J, Marmot M (2005) Positive affect and healthrelated neuroendocrine, cardiovascular, and inflammatory processes. PNAS 102(18):6508-6512

Taylor S, Klein L, Lewis B, Gruenewald T, Gurung R, Updegraff J (2000) Biobehavioral responses to stress in females: tend-andbefriend, not fight-or-flight. Psychol Rev 107(3):411-429

Tirindelli R, Dibattista M, Pifferi S, Menini A (2009) From Pheromones to Behaviour Physiological Review 89:921-956

Van Kleef G, A. (2009) How emotions regulate social life: the emotions as social information (EASI) model. Curr Dir Psychol Sci 18:184-188

Waugh C, Fredrickson B (2006) Nice to know you: positive emotions, self-other overlap, and complex understanding in the formation of a new relationship. Journal of Positive Psychology 1(2):93-106

Publisher's Note Springer Nature remains neutral with regard to jurisdictional claims in published maps and institutional affiliations. 